Volume 136, Number 1, January 2008, Pages 55-60

S 0002-9939(07)08944-7

Article electronically published on September 25, 2007

\title{
SUBMANIFOLDS OF REAL ALGEBRAIC VARIETIES
}

\author{
W. KUCHARZ
}

(Communicated by Paul Goerss)

\begin{abstract}
By the Nash-Tognoli theorem, each compact smooth manifold $M$ is diffeomorphic to a nonsingular real algebraic set, called an algebraic model of $M$. We construct algebraic models $X$ of $M$ with controlled behavior of the group of cohomology classes represented by algebraic subsets of $X$.
\end{abstract}

\section{INTRODUCTION}

A compact smooth (of class $C^{\infty}$ ) manifold is said to be a boundary if it is diffeomorphic to the boundary of a compact smooth manifold with boundary. By convention, the empty manifold is a boundary. The goal of the present paper is to demonstrate that boundaries play a surprisingly interesting role in real algebraic geometry (cf. [4, Theorem 1.4] for the first result of this kind).

All real algebraic varieties in this paper are assumed to be affine (that is, isomorphic to an algebraic subset of $\mathbb{R}^{n}$ for some $n$ ). For background material on real algebraic geometry the reader may consult [2]. Every real algebraic variety carries also the Euclidean topology, induced by the usual metric topology on $\mathbb{R}$. Unless explicitly stated otherwise, all topological notions related to real algebraic varieties will refer to the Euclidean topology.

Given a compact nonsingular real algebraic variety $X$, we denote by $H_{d}^{a l g}(X, \mathbb{Z} / 2)$ the subgroup of $H_{d}(X, \mathbb{Z} / 2)$ of homology classes represented by $d$-dimensional algebraic subsets of $X$; cf. $[1,2,3,5]$. For technical reasons it is more convenient to work with cohomology groups. We set

$$
H_{\text {alg }}^{c}(X, \mathbb{Z} / 2):=D_{X}^{-1}\left(H_{d}^{\text {alg }}(X, \mathbb{Z} / 2)\right),
$$

where $c+d=\operatorname{dim} X$ and $D_{X}: H^{c}(X, \mathbb{Z} / 2) \rightarrow H_{d}(X, \mathbb{Z} / 2)$ is the Poincaré duality isomorphism. The groups $H_{\text {alg }}^{c}(-, \mathbb{Z} / 2)$ are of fundamental interest in real algebraic geometry and will also be in the center of our attention here. Their basic properties and applications are surveyed in [3].

Let $M$ be a compact smooth manifold of dimension $m$. Denote by $[M]$ the fundamental class of $M$ in $H_{m}(M, \mathbb{Z} / 2)$. For any smooth $d$-dimensional submanifold $N$ of $M$ (submanifolds are always assumed to be closed subsets), we let $[N]_{M}$ denote the homology class in $H_{d}(M, \mathbb{Z} / 2)$ represented by $N$. We set $[N]_{M}=0$ if $N$ is empty.

Received by the editors September 10, 2005 and, in revised form, September 28, 2006. 2000 Mathematics Subject Classification. Primary 14P05, 14C25. 
Given a finite collection $\mathcal{F}$ of smooth submanifolds of $M$, we define

$$
\begin{aligned}
G^{k}(\mathcal{F}):= & \left\{u \in H^{k}(M, \mathbb{Z} / 2) \mid\left\langle u,[N]_{M}\right\rangle=0\right. \text { for all } \\
& N \text { in } \mathcal{F} \text { with } \operatorname{dim} N=k\} .
\end{aligned}
$$

Here, as usual, $\langle$,$\rangle stands for scalar product (Kronecker index). If there is no$ $k$-dimensional submanifold in $\mathcal{F}$, then $G^{k}(\mathcal{F}):=H^{k}(M, \mathbb{Z} / 2)$. Clearly, $G^{k}(\mathcal{F})$ is a subgroup of $H^{k}(M, \mathbb{Z} / 2)$.

Recall that $\mathcal{F}$ is said to be in general position if for each point $x$ in $M$, the collection $\mathcal{F}_{x}=\{N \in \mathcal{F} \mid x \in N\}$ is either empty or otherwise $\operatorname{codim}\left(\bigcap_{N \in \mathcal{F}_{x}} T_{x} N\right)=$ $\sum_{N \in \mathcal{F}_{x}} \operatorname{codim} T_{x} N$, where $T_{x} N$ is regarded as a subspace of the tangent space $T_{x} M$. If $\mathcal{F}$ is in general position and $N_{1}, \ldots, N_{s}$ are in $\mathcal{F}$, then $N_{1} \cap \ldots \cap N_{s}$ is a smooth submanifold of $M$.

Definition 1.1. A finite collection $\mathcal{F}$ of smooth submanifolds of $M$ is said to be admissible if:

(i) $\operatorname{dim} N<\operatorname{dim} M$ for all $N$ in $\mathcal{F}$;

(ii) $\mathcal{F}$ is in general position;

(iii) if $N_{1}, \ldots, N_{s}$ are in $\mathcal{F}$, then $N_{1} \cap \ldots \cap N_{s}$ is in $\mathcal{F}$;

(iv) each submanifold in $\mathcal{F}$ has trivial normal vector bundle.

If $M^{\prime}$ is another smooth manifold and if $\varphi: M^{\prime} \rightarrow M$ is a smooth diffeomorphism, then

$$
\varphi^{*} \mathcal{F}:=\left\{\varphi^{-1}(N) \mid N \in \mathcal{F}\right\}
$$

is a collection of smooth submanifolds of $M^{\prime}$. The collection $\varphi^{*} \mathcal{F}$ is admissible, provided $\mathcal{F}$ is admissible.

Theorem 1.2. Let $X$ be a compact nonsingular real algebraic variety and let $\mathcal{F}$ be an admissible collection of smooth submanifolds of $X$. If

$$
H_{\text {alg }}^{k}(X, \mathbb{Z} / 2) \subseteq G^{k}(\mathcal{F})
$$

for some nonnegative integer $k$, then each $k$-dimensional manifold in $\mathcal{F}$ is a boundary.

Theorem 1.2 is easy to prove. Its purpose is to provide motivation for our main result, Theorem 1.3, below. By Tognoli's theorem [14] (cf. also [2, Theorem 14.1.10] and, for a weaker but influential result, [12]), each compact smooth manifold $M$ is diffeomorphic to a nonsingular real algebraic variety, called an algebraic model of $M$. Constructing algebraic models satisfying some additional desirable conditions is an interesting and active area of research; $\mathrm{cf}$. $[1,2,3,8,9]$.

Theorem 1.3. Let $M$ be a compact smooth manifold and let $\mathcal{F}$ be an admissible collection of smooth submanifolds of $M$. If each manifold in $\mathcal{F}$ is a boundary, then there exist an irreducible algebraic model $X$ of $M$ and a smooth diffeomorphism $\varphi: X \rightarrow M$ such that

$$
H_{a l g}^{k}(X, \mathbb{Z} / 2) \subseteq G^{k}\left(\varphi^{*} \mathcal{F}\right)
$$

for all nonnegative integers $k$.

Theorem 1.3 is useful for constructing examples such that $H_{\text {alg }}^{k}(-, \mathbb{Z} / 2) \neq$ $H^{k}(-, \mathbb{Z} / 2)$. 
Example 1.4. The $n$-fold product $T^{n}=S^{1} \times \cdots \times S^{1}$ of the unit circle $S^{1}$ has an algebraic model $X$ with $H_{\text {alg }}^{k}(X, \mathbb{Z} / 2) \neq H^{k}(X, \mathbb{Z} / 2)$ for $1 \leq k \leq n-1$. Indeed, suppose $n \geq 2$, since for $n=1$ the assertion is void. Choose $n-1$ distinct points $p_{1}, \ldots, p_{n-1}$ in $S^{1}$ and set

$$
N_{i}=\left\{\left(x_{1}, \ldots, x_{n}\right) \in T^{n} \mid x_{1}=\cdots=x_{i}=p_{i}\right\}
$$

for $1 \leq i \leq n-1$. Then $\mathcal{F}:=\left\{N_{1}, \ldots, N_{n-1}\right\}$ is an admissible collection of smooth submanifolds of $T^{n}$. Moreover, $\operatorname{codim}_{T^{n}} N=i$ and $N_{i}$ is a boundary for $1 \leq i \leq n-1$. Hence the assertion follows from Theorem 1.3.

Theorems 1.2 and 1.3 will be proved in the next section.

\section{ProOfS}

As usual, the $i$ th Stiefel-Whitney class of a smooth manifold $M$ will be denoted by $w_{i}(M)$. Given a smooth submanifold $N$ of $M$, we set

$$
[N]^{M}:=D_{M}^{-1}\left([N]_{M}\right) \text {. }
$$

Henceforth, both the homology class $[N]_{M}$ and cohomology class $[N]^{M}$ will be used. Basic properties of the products $\langle\rangle,, \cap, \cup$ familiar from algebraic topology [6] will be used without further explanation.

Lemma 2.1. Let $M$ be a compact smooth manifold and let $N$ be a $k$-dimensional smooth submanifold of $M$. If the normal vector bundle of $N$ in $M$ is trivial, then the following conditions are equivalent:

(a) $N$ is a boundary,

(b) $\left\langle w_{i_{1}}(M) \cup \ldots \cup w_{i_{r}}(M),[N]_{M}\right\rangle=0$ for all nonnegative integers $i_{1}, \ldots, i_{r}$ with $i_{1}+\cdots+i_{r}=k$,

(c) $\left\langle w_{i_{1}}(M) \cup \ldots \cup w_{i_{r}}(M) \cup[N]^{M},[M]\right\rangle=0$ for all nonnegative integers $i_{1}, \ldots, i_{r}$ with $i_{1}+\cdots+i_{r}=k$.

Proof. Let $e: N \hookrightarrow M$ be the inclusion map. Then

$$
[N]^{M} \cap[M]=D_{M}\left([N]^{M}\right)=[N]_{M}=e_{*}([N]) .
$$

Setting $w=w_{i_{1}}(M) \cup \ldots \cup w_{i_{r}}(M)$, we have

$$
\begin{aligned}
\left\langle w \cup[N]^{M},[M]\right\rangle & =\left\langle w,[N]^{M} \cap[M]\right\rangle \\
& =\left\langle w,[N]_{M}\right\rangle \\
& =\left\langle w, e_{*}([N])\right\rangle \\
& =\left\langle e^{*}(w),[N]\right\rangle \\
& =\left\langle e^{*}\left(w_{i_{1}}(M)\right) \cup \ldots \cup e^{*}\left(w_{i_{r}}(M)\right),[N]\right\rangle \\
& =\left\langle w_{i_{1}}(N) \cup \ldots \cup w_{i_{r}}(N),[N]\right\rangle,
\end{aligned}
$$

where the last equality holds since the triviality of the normal vector bundle of $N$ implies that $e^{*}\left(w_{i}(M)\right)=w_{i}(N)$ for all $i \geq 0$. The proof is complete since, by [13, Théorèmes IV.3, IV.10], $N$ is a boundary if and only if $\left\langle w_{i_{1}}(N) \cup \ldots \cup w_{i_{r}}(N),[N]\right\rangle=$ 0 for all nonnegative integers $i_{1}, \ldots, i_{r}$ with $i_{1}+\cdots+i_{r}=k$.

Proof of Theorem 1.2. Every cup product $w_{i_{1}}(X) \cup \ldots \cup w_{i_{r}}(X)$ is in $H_{\text {alg }}^{k}(X, \mathbb{Z} / 2)$, where $k=i_{1}+\cdots+i_{r}$; cf. $[1,2,3,5]$. By assumption, for any $k$-dimensional submanifold $N$ in $\mathcal{F}$, we have

$$
\left\langle w_{i_{1}}(X) \cup \ldots \cup w_{i_{r}}(X),[N]_{X}\right\rangle=0,
$$

which completes the proof in view of Lemma 2.1. 
The proof of Theorem 1.3 requires further preparations. Given a smooth manifold $P$, let $\mathfrak{N}_{*}(P)$ denote the unoriented bordism group of $P$; cf. [7]. The following fundamental result will play a crucial role.

Theorem 2.2. Let $P$ be a smooth manifold. Two smooth maps $f: M \rightarrow P$ and $g: N \rightarrow P$, where $M$ and $N$ are compact smooth manifolds of dimension $d$, represent the same bordism class in $\mathfrak{N}_{*}(P)$ if and only if for every nonnegative integer $q$ and every cohomology class $v$ in $H^{q}(P, \mathbb{Z} / 2)$, one has

$$
\left\langle w_{i_{1}}(M) \cup \ldots \cup w_{i_{r}}(M) \cup f^{*}(v),[M]\right\rangle=\left\langle w_{i_{1}}(N) \cup \ldots \cup w_{i_{r}}(N) \cup g^{*}(v),[N]\right\rangle
$$

for all nonnegative integers $i_{1}, \ldots, i_{r}$ with $i_{1}+\cdots+i_{r}=d-q$.

Reference for the proof. [7, (17.3)].

If $W$ is a nonsingular real algebraic variety, then a bordism class in $\mathfrak{N}_{*}(W)$ is said to be algebraic if it can be represented by a regular map $h: Y \rightarrow W$, where $Y$ is a compact nonsingular real algebraic variety.

We will also make use of a certain construction from real algebraic geometry. Let $X$ be a compact nonsingular real algebraic variety. Define $A l g^{l}(X)$ to be the subset of $H^{l}(X, \mathbb{Z} / 2)$ that consists of all elements $v$ for which there exist a compact irreducible nonsingular real algebraic variety $T$ (depending on $v$ ), two points $t_{0}$ and $t_{1}$ in $T$, and a cohomology class $z$ in $H_{\text {alg }}^{l}(X \times T, \mathbb{Z} / 2)$ such that

$$
v=i_{t_{1}}^{*}(z)-i_{t_{0}}^{*}(z) \text {. }
$$

Here given $t$ in $T$, we let $i_{t}: X \rightarrow X \times T$ denote the map defined by $i_{t}(x)=(x, t)$ for all $x$ in $X$. For properties and an alternative definition of $A l g^{l}(-)$, the reader may refer to $[10,11]$. Below we will need the following facts. The set $A^{l} g^{l}(X)$ is a subgroup of $H_{\text {alg }}^{l}(X, \mathbb{Z} / 2)$, which has the expected functorial property: if $f: X \rightarrow Y$ is a regular map between compact nonsingular real algebraic varieties, then the induced homomorphism $f^{*}: H^{l}(Y, \mathbb{Z} / 2) \rightarrow H^{l}(X, \mathbb{Z} / 2)$ satisfies

$$
f^{*}\left(A l g^{l}(Y)\right) \subseteq A l g^{l}(X) ;
$$

cf. [1, p. 114].

Proposition 2.3. Let $X$ be a compact nonsingular real algebraic variety. If $u$ is in $H_{\text {alg }}^{k}(X, \mathbb{Z} / 2)$ and $v$ is in $A^{l} g^{l}(X)$, where $k+l=\operatorname{dim} X$, then $\langle u \cup v,[X]\rangle=0$.

References for the proof. [10, Theorem 2.1] (cf. also [11, Theorem 4.4]).

Example 2.4. Given a positive integer $l$ let $B^{l}$ be an irreducible nonsingular real algebraic variety with precisely two connected components $B_{0}^{l}$ and $B_{1}^{l}$, each diffeomorphic to the unit $l$-sphere $S^{l}$ (for example $B^{l}=\left\{\left(x_{0}, \ldots, x_{l}\right) \in \mathbb{R}^{l+1} \mid x_{0}^{4}-\right.$ $\left.\left.4 x_{0}^{2}+1+x_{1}^{2}+\cdots+x_{l}^{2}=0\right\}\right)$. Let $B=B^{l_{1}} \times \cdots \times B^{l_{s}}$ and $B_{0}=B_{0}^{l_{1}} \times \cdots \times B_{0}^{l_{s}}$. Then

$$
H^{q}\left(B_{0}, \mathbb{Z} / 2\right)=\delta^{*}\left(H^{q}(B, \mathbb{Z} / 2)\right)=\delta^{*}\left(A l g^{q}(B)\right)
$$

for all $q \geq 0$, where $\delta: B_{0} \hookrightarrow B$ is the inclusion map. This assertion is a minor generalization of [11, Example 4.5].

For convenience we introduce the following notation. For any finite collection $\mathcal{F}$ of smooth submanifolds of a compact smooth manifold $M$, denote by $A^{l}(\mathcal{F})$ the subgroup of $H^{l}(M, \mathbb{Z} / 2)$ generated by $\left\{[N]^{M} \mid N \in \mathcal{F}, \operatorname{codim}_{M} N=l\right\}$. If $k+l=\operatorname{dim} M$, then

$$
G^{k}(\mathcal{F})=\left\{u \in H^{k}(M, \mathbb{Z} / 2) \mid\langle u \cup v,[M]\rangle=0 \text { for all } v \text { in } A^{l}(\mathcal{F})\right\} .
$$


This follows immediately from the equality $\langle u \cup v,[M]\rangle=\left\langle u, D_{M}(v)\right\rangle$.

Proof of Theorem 1.3. Observe that $G^{0}(\mathcal{F})$ contains the subgroup of $H^{0}(M, \mathbb{Z} / 2)$ generated by 1 . On the other hand, if $Y$ is a compact irreducible nonsingular real algebraic variety, then $H_{\text {alg }}^{0}(Y, \mathbb{Z} / 2)$ is the subgroup of $H^{0}(Y, \mathbb{Z} / 2)$ generated by 1 . Hence, without loss of generality, we can enlarge the collection $\mathcal{F}$ by adding to it finitely many 0-dimensional submanifolds of $M$, each consisting of an even number of points. Thus we can assume that $\mathcal{F}$ has the following property: if $M_{1}$ and $M_{2}$ are distinct connected components of $M$, then $\left\{x_{1}, x_{2}\right\}$ is in $\mathcal{F}$ for some points $x_{1}$ in $M_{1}$ and $x_{2}$ in $M_{2}$.

Let $\mathcal{F}=\left\{N_{1}, \ldots, N_{s}\right\}$ and $l_{i}=\operatorname{codim}_{M} N_{i}$ for $1 \leq i \leq s$. Note that $l_{i} \geq 1$. We will use notation introduced in Example 2.4. There is a smooth map $f_{i}: M \rightarrow$ $B^{l_{i}}$ such that $f_{i}(M) \subseteq B_{0}^{l_{i}}$ and $f_{i}^{*}\left(H^{l_{i}}\left(B^{l_{i}}, \mathbb{Z} / 2\right)\right)$ is the subgroup of $H^{l_{i}}(M, \mathbb{Z} / 2)$ generated by $\left[N_{i}\right]^{M}$. This assertion follows from a well-known fact that if the normal vector bundle of a smooth submanifold $N$ of $M$ is trivial and $\operatorname{codim}_{M} N=$ $l \geq 1$, then one can find a smooth map $h: M \rightarrow S^{l}$ with $h^{*}(\lambda)=[N]^{M}$, where $\lambda$ is the unique generator of $H^{l}\left(S^{l}, \mathbb{Z} / 2\right) \cong \mathbb{Z} / 2$; cf. [13, Théorème II.2]. Setting $f=\left(f_{1}, \ldots, f_{s}\right): M \rightarrow B=B^{l_{1}} \times \cdots \times B^{l_{s}}$, we have

$$
f^{*}\left(H^{q}(B, \mathbb{Z} / 2)\right)=A^{q}(\mathcal{F}) \quad \text { for all } q \geq 1 .
$$

Let $c: M \rightarrow B$ be a constant map, whose single value is a point in $B_{0}$. Since $M$ has an algebraic model, the bordism class of $c$ in $\mathfrak{N}_{*}(B)$ is algebraic. We claim that for every nonnegative integer $q$ and every cohomology class $b$ in $H^{q}(B, \mathbb{Z} / 2)$,

$(2)\left\langle w_{i_{1}}(M) \cup \ldots \cup w_{i_{r}}(M) \cup f^{*}(b),[M]\right\rangle=\left\langle w_{i_{1}}(M) \cup \ldots \cup w_{i_{r}}(M) \cup c^{*}(b),[M]\right\rangle$

for all nonnegative integers $i_{1}, \ldots, i_{r}$ with $i_{1}+\cdots+i_{r}=\operatorname{dim} M-q$. The claim is obvious if $q=0$. For $q \geq 1$ condition (2) is equivalent to

$$
\left\langle w_{i_{1}}(M) \cup \ldots \cup w_{i_{r}}(M) \cup f^{*}(b),[M]\right\rangle=0 .
$$

The last equality holds in view of (1) and Lemma 2.1, and hence (2) is proved.

It follows from (2) and Theorem 2.2 that $f$ and $c$ represent the same bordism class in $\mathfrak{N}_{*}(B)$. In particular, the bordism class of $f$ in $\mathfrak{N}_{*}(B)$ is algebraic. By $[1$, Theorem 2.8.4], there exist an algebraic model $X$ of $M$, a smooth diffeomorphism $\varphi: X \rightarrow M$, and a regular map $g: X \rightarrow B$ such that $g$ is homotopic to $f \circ \varphi$. It remains to show that $X$ and $\varphi$ have the properties stated in the theorem. This can be done as follows. Since $g$ is homotopic to $f \circ \varphi$, we get $\varphi^{*} \circ f^{*}=(f \circ \varphi)^{*}=g^{*}$ in cohomology, and hence for all $q \geq 0$,

$$
\varphi^{*}\left(f^{*}\left(H^{q}(B, \mathbb{Z} / 2)\right)\right)=g^{*}\left(H^{q}(B, \mathbb{Z} / 2)\right)=g^{*}\left(A l g^{q}(B)\right),
$$

where the last equality follows from the inclusion $g(X) \subseteq B_{0}$ and Example 2.4. We also have

$$
g^{*}\left(A l g^{q}(B)\right) \subseteq A l g^{q}(X),
$$

the map $g: X \rightarrow B$ being regular. Conditions (1), (3), and (4) combined yield

$$
\varphi^{*}\left(A^{q}(\mathcal{F})\right) \subseteq A l g^{q}(X) \text { for all } q \geq 1 .
$$

Let $k$ and $l$ be integers satisfying $k \geq 0, l \geq 0$, and $k+l=m$, where $m=$ $\operatorname{dim} M=\operatorname{dim} X$. Since $\varphi^{*}\left(A^{l}(\mathcal{F})\right)=A^{l}\left(\varphi^{*} \mathcal{F}\right)$ and

$$
G^{k}\left(\varphi^{*} \mathcal{F}\right)=\left\{u \in H^{k}(X, \mathbb{Z} / 2) \mid\langle u \cup v,[X]\rangle=0 \text { for all } v \in A^{l}\left(\varphi^{*} \mathcal{F}\right)\right\},
$$


it follows from (5) and Proposition 2.3 that

$$
H_{\text {alg }}^{k}(X, \mathbb{Z} / 2) \subseteq G^{k}\left(\varphi^{*} \mathcal{F}\right)
$$

for $0 \leq k \leq m-1$. In fact (6) holds for all $k \geq 0$, the equality $G^{k}\left(\varphi^{*} \mathcal{F}\right)=$ $H^{k}(X, \mathbb{Z} / 2)$ being automatically satisfied if $k \geq m$.

In order to complete the proof, we need to demonstrate that $X$ is irreducible. This is obvious if $M$ is connected. Suppose then that $M$ is disconnected and $X$ is reducible. Let $X_{1}$ and $X_{2}$ be distinct irreducible components of $X$. There are points $p_{1}$ in $X_{1}$ and $p_{2}$ in $X_{2}$ such that $P=\left\{p_{1}, p_{2}\right\}$ is in $\varphi^{*} \mathcal{F}$ (see the beginning of the proof). In particular, $[P]^{X}$ belongs to $A^{m}\left(\varphi^{*} \mathcal{F}\right)=\varphi^{*}\left(A^{m}(\mathcal{F})\right)$, which in view of (5) implies that $[P]^{X}$ is in $A l g^{m}(X)$. If $e: X_{1} \hookrightarrow X$ is the inclusion map, then $e^{*}\left(A l g^{m}(X)\right) \subseteq A l g^{m}\left(X_{1}\right)$, and hence $e^{*}\left([P]^{X}\right)=\left[\left\{p_{1}\right\}\right]^{X_{1}}$ is in $A l g^{m}\left(X_{1}\right)$. However, $\left\langle\left[\left\{p_{1}\right\}\right]^{X_{1}},\left[X_{1}\right]\right\rangle=1$, which contradicts Proposition 2.3. Thus $X$ is irreducible, as required.

\section{REFERENCES}

[1] S. Akbulut and H. King, Topology of Real Algebraic Sets, Math. Sci. Research Institute Publ. 25, Springer, 1992. MR1225577 (94m:57001)

[2] J. Bochnak, M. Coste and M.-F. Roy, Real Algebraic Geometry, Ergebnisse der Math. und ihrer Grenzgeb. Folge (3), Vol. 36, Springer, 1998. MR1659509 (2000a:14067)

[3] J. Bochnak and W. Kucharz, On homology classes represented by real algebraic varieties, Banach Center Publications 44 (1998), 21-35. MR1677394 (2000b:14080)

[4] J. Bochnak and W. Kucharz, On approximation of smooth submanifolds by nonsingular real algebraic subvarieties, Ann. Sci. Ec. Norm. Sup. 36 (2003), 685-690. MR2032984 (2004j:14063)

[5] A. Borel and A. Haefliger, La classe d'homologie fondamentale d'un espace analytique, Bull. Soc. Math. France 89 (1961), 461-513. MR0149503 (26:6990)

[6] G. Bredon, Topology and Geometry, New York, Berlin, Heidelberg, Springer, 1997. MR1700700 (2000b:55001)

[7] P. Conner, Differentiable Periodic Maps, Lecture Notes in Math. Vol. 738, Springer, 1979. MR548463 (81f:57018)

[8] J. Huisman and F. Mangolte, Every orientable Seifert 3-manifold is a real component of a uniruled algebraic variety, Topology 44 (2005), 63-71. MR2104001 (2005h:14139)

[9] J. Kollár, The topology of real and complex algebraic varieties, Taniguchi Conference on Mathematics, Nara 1998, Advanced Studies in Pure Mathematics, Vol. 31, Mathematical Society of Japan, Tokyo, 2001, pp. 127-145. MR1865090 (2003a:14033)

[10] W. Kucharz, Algebraic equivalence and homology classes of algebraic cycles, Math. Nachr. 180 (1996), 135-140. MR1397672 (97e:14009)

[11] W. Kucharz, Algebraic cycles and algebraic models of smooth manifolds, J. Algebraic Geometry 11 (2002), 101-127. MR1865915 (2002j:14065)

[12] J. Nash, Real algebraic manifolds, Ann. of Math. 56 (1952), 405-421. MR0050928 (14:403b)

[13] R. Thom, Quelques propriétés globales des variétés différentiables, Comment. Math. Helv. 28 (1954), 17-86. MR0061823 (15:890a)

[14] A. Tognoli, Su una congettura di Nash, Ann. Scuola Norm. Sup. Pisa, Sci. Fis. Mat. 27 (3) (1973), 167-185. MR0396571 (53:434)

Department of Mathematics and Statistics, University of New Mexico, Albuquerque, New Mexico 87131-1141

E-mail address: kucharz@math.unm.edu 